Revue des patrimoines

16 | 2011

Le patrimoine militaire et la question urbaine

\title{
La base sous-marine de Bordeaux, sous le béton la culture
}

Mathieu Marsan

\section{CpenEdition}

Journals

\section{Édition électronique}

URL : http://journals.openedition.org/insitu/9526

DOI : 10.4000/insitu.9526

ISSN : 1630-7305

\section{Éditeur}

Ministère de la culture

Référence électronique

Mathieu Marsan, «La base sous-marine de Bordeaux, sous le béton la culture », In Situ [En ligne], 16 | 2011, mis en ligne le 20 février 2013, consulté le 19 avril 2019. URL : http:// journals.openedition.org/insitu/9526; DOI : 10.4000/insitu.9526

Ce document a été généré automatiquement le 19 avril 2019

\section{(c) $($ i) $(9)$}

In Situ Revues des patrimoines est mis à disposition selon les termes de la licence Creative Commons Attribution - Pas d'Utilisation Commerciale - Pas de Modification 4.0 International. 


\title{
La base sous-marine de Bordeaux, sous le béton la culture
}

\author{
Mathieu Marsan
}

1 La base sous-marine de Bordeaux est l'une des cinq bases construites sur le territoire français par les Allemands au cours de la Seconde Guerre mondiale, elle est à inclure dans l'ensemble hétérogène du Mur de l'Atlantique ${ }^{1}$. Cette fortification était considérée par Adolf Hitler (1889-1945) comme un véritable outil de propagande du III ${ }^{\mathrm{e}}$ Reich. Le Führer avait imaginé qu'elle durerait mille ans, bien que les travaux engagés en 1940 n'arrivèrent jamais à leur terme. L'occupation du littoral français par les troupes nazies se traduit dans les grands ports par la construction de gigantesques abris bétonnés destinés à la protection de la précieuse force sous-marine italo-allemande. Entre décembre 1940 et avril 1941, les chantiers supervisés par l'Ot-Einsatzgruppe West, branche de l'Organisation TODT affiliée à la France, et la Kriegsmarine sont initiés dans les ports stratégiques de Brest, Lorient, Saint-Nazaire et La Rochelle. À Bordeaux, les travaux débutent plus tardivement en septembre 1941. L'emplacement du port et ses bassins à flot situés au nord de la ville dans le quartier populaire de Bacalan paraît alors évident. En effet, depuis l'été 1940 , des sous-marins italiens stationnent dans le bassin à flot $n^{\circ} 1$ qui constitue alors, la première base bordelaise appelée « Betasom ${ }^{2}$ ». La base sous-marine s'enracine ainsi à l'emplacement du réservoir d'alimentation du bassin à flot $n^{\circ} 2$, destiné à devenir le bassin à flot $\mathrm{n}^{\circ} 3$, à l'aide de trois mille poutres en béton armé assurant sa stabilité dans le sol tourbeux (fig. $\left.\mathbf{n}^{\circ} \mathbf{1}\right)$. 


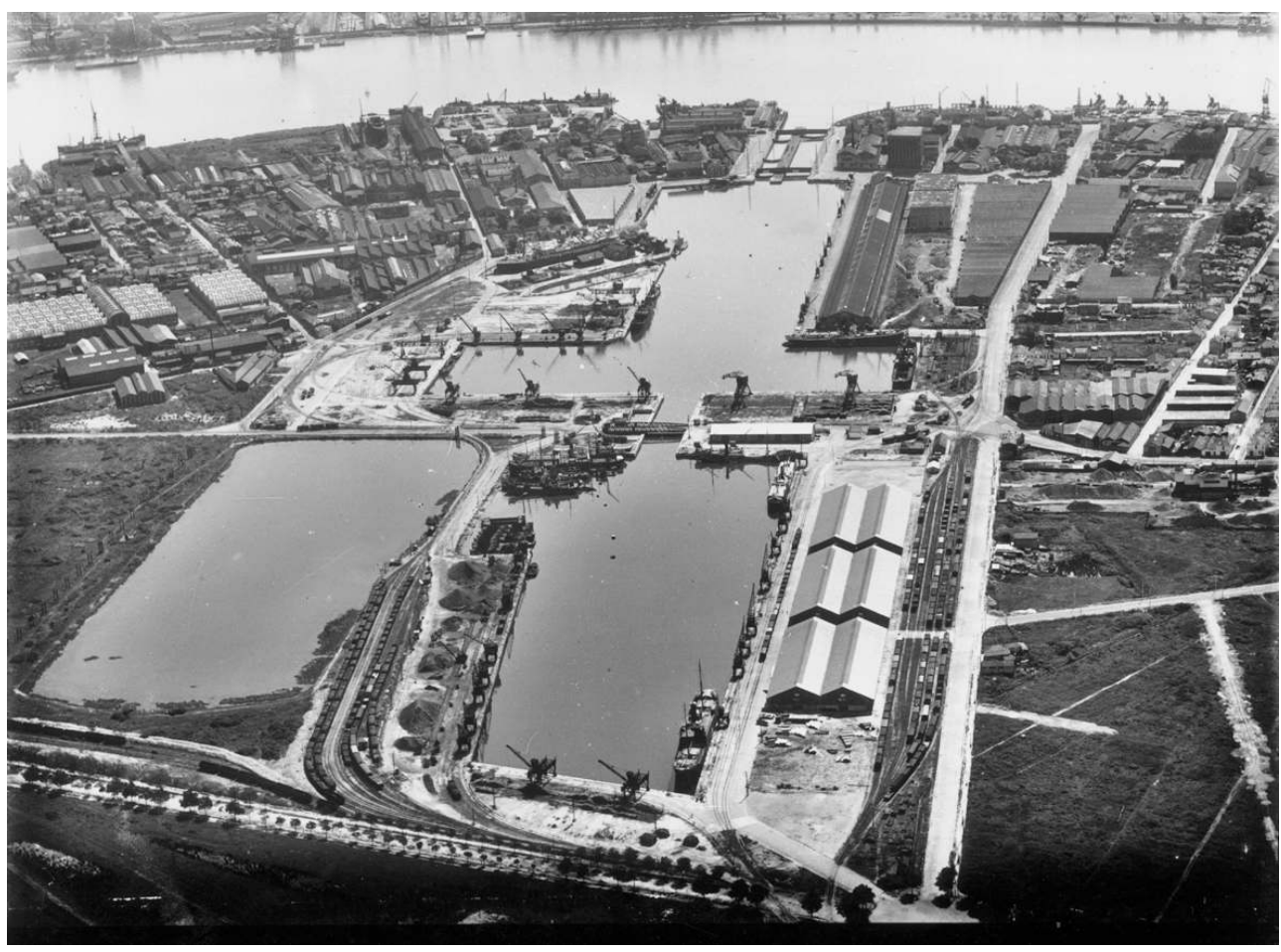

Vue aérienne des bassins à flot $n^{\circ} 1$ et $n^{\circ} 2$ et du bassin d'alimentation, à l'emplacement de la future base sous-marine, 1924.

(c) Archives de la Mémoire de Bordeaux.

2 En dix-neuf mois de travaux, six cent mille mètres cubes de béton sont coulés grâce au travail constant fourni par six mille cinq cents ouvriers - volontaires, contractuels ou forcés - afin de rendre la base opérationnelle. À l'achèvement des travaux en mai 1943, ses dimensions en traduisent les ambitions démesurées, elle occupe une surface au sol de quarante-cinq mille mètres carrés, soit deux cent trente-cinq mètres de long, cent soixante mètres de large pour une hauteur de dix-neuf mètres. Il faut ajouter à cela une tour annexe, qui lui est accolée pour les machineries et les bureaux, mesurant soixantequinze mètres de longueur, soixante mètres de largeur et vingt-trois mètres de haut. Le plan de la base sous-marine de Bordeaux produit par le service Marinebauwesen de la Kriegsmarine reprend ceux des bases de Brest, de Saint-Nazaire et de La Pallice et comprend onze alvéoles de trois types différents ${ }^{3}$. Les alvéoles 1 à 4 sont les plus imposantes, uniquement utilisables à flot, elles peuvent respectivement accueillir deux sous-marins. Les quatre alvéoles suivantes (5 à 8) présentent une surface moins conséquente, mais peuvent être utilisées en tant que cales sèches grâce à des pompes de vidange et d'assèchement nécessaire à l'accueil d'un sous-marin pouvant subir des modifications d'envergures. Enfin, les trois dernières, les alvéoles 9 à 11, placées en retrait par rapport aux huit précédentes, présentent de faibles dimensions leur permettant d'abriter un sous-marin chacune dans un espace transformable en cale sèche. $\mathrm{Au}$ total, quinze sous-marins pouvaient trouver refuge à l'intérieur de la base sousmarine afin de procéder à des travaux d'entretien et de réparation tout en étant isolés du bassin à flot $n^{\circ} 2$ par des bateaux-portes s'encastrant à l'entrée de chacune des alvéoles et par des rideaux mobiles à double blindage en acier. Des zones techniques nommées «cellules», situées dans le prolongement des alvéoles, permettaient le stockage du 
matériel nécessaire à la maintenance et au ravitaillement des submersibles. Par ailleurs, la base sous-marine de Bordeaux diffère de ses aînées par la constitution de son toit. Afin de protéger l'édifice de potentiels bombardements, deux dalles de béton, d'une épaisseur totale de 5,60 m, ont été coulées au gré des travaux et complétées par une troisième structure de type Fangrost destinée à déclencher au premier contact l'explosion des bombes avant qu'elles n'atteignent le toit ${ }^{4}$. Enfin, trois bunkers majeurs se trouvaient à proximité de la base de Bacalan, au nord, la soute à torpille et la citerne à fioul respectivement liées par un réseau ferré et un oléoduc souterrain au U-Bunker et au sudest, une écluse couverte afin de protéger l'écoulement des eaux et l'accès des sous-marins aux bassins à flot (fig. $\left.\mathbf{n}^{\circ} \mathbf{2}\right)$.

Figure 2

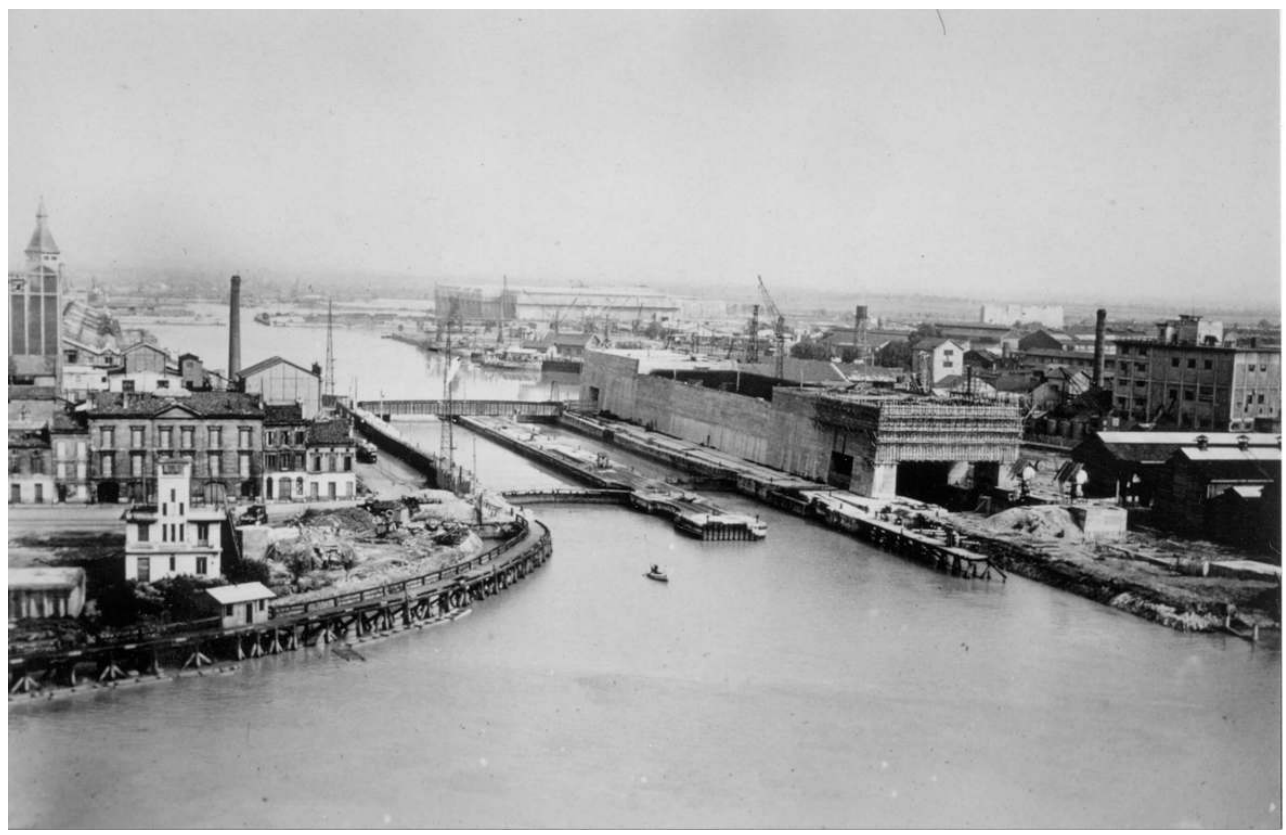

Vue des écluses donnant accès aux bassins à flot. Au premier plan, l'écluse couverte en cours de construction, en arrière-plan, la base sous-marine et la citerne à fioul, 1944

(c) La Mémoire de Bordeaux/Archives Grand Port Maritime de Bordeaux.

Cet ensemble est à plusieurs reprises la cible des bombardements alliés, dégradant faiblement la base sous-marine mais causant la mort de plusieurs centaines de riverains. Contrairement à l'écluse couverte et à la soute à torpilles détruites à la Libération, la base sous-marine et la citerne à fioul, aujourd'hui située sur un terrain privé, sont conservées. Cette étape marque le début d'une longue période de déshérence pour cet indestructible vestige de l'Occupation qui devient rapidement le terrain de chasse favori des ferrailleurs ainsi qu'un lieu incontournable pour la jeunesse bordelaise en mal de sensations fortes. En 1945, la Marine Nationale confie la base sous-marine au Port Autonome de Bordeaux, dénommé Grand Port Maritime de Bordeaux depuis octobre 2008. Celle-ci devient alors un espace capable de stimuler tous les fantasmes, sans pour autant engendrer un programme complet de réhabilitation. 


\section{Un bunker en marge de la ville}

\section{Des expérimentations annonciatrices. Une improbable reconversion}

4 L'architecture de la base sous-marine de Bordeaux a pour origine le dessin d'un ingénieur. Ses formes coulées dans le béton répondent avec brio à l'unique fonction d'un édifice militaire : protéger ses occupants des attaques extérieures. Un ennemi inattendu, le temps, a toutefois patiné ses murs, l'a rendu sauvage, permettant à la nature d'y reprendre ses droits après la Libération. C'est pourtant par la figure d'un homme ayant vécu l'épisode douloureux des camps de concentration que la base sous-marine de Bordeaux connaît sa première « invasion culturelle ». En 1964, Jean Cayrol, réputé pour sa poétique "lazaréenne", explore notamment les phénomènes de concentration et d'identification au travers de son second long métrage Le Coup de grâce, dont la scène finale se situe dans la base bordelaise ${ }^{5}$. D'élément de décor, le U-Bunker se transforme en scène de théâtre lors de la quatorzième édition du festival Sigma en 1978 sous l'impulsion de son créateur Roger Lafosse. Ce dernier a su tirer partie du potentiel visuel et émotionnel du lieu malgré le fait qu'il ait connu la base durant l'Occupation et la considère comme un lieu abominable ${ }^{6}$. La présentation de la pièce de Novalis, produite par Jacques Albert-Canque en collaboration avec le Groupe 33, intitulée Pour en finir avec la fleur bleue, correspond à la première ouverture au public de la base sous-marine de Bordeaux ${ }^{7}$. Lors des éditions suivantes, l'utilisation de l'espace intérieur se fait plus rare, Roger Lafosse préférant implanter les spectacles humoristiques de Royal de Luxe à l'ombre du bunker. Malgré un vif intérêt de la part de la scène artistique émergeante de Bordeaux entre les années 1960 et 1980, la base sous-marine reste toujours à l'abandon. Un abandon doublement justifié par l'importance des coûts d'entretien et de gestion d'un tel bâtiment par le Port Autonome de Bordeaux. C'est pourtant ce climat qui suscite l'intérêt du peintre et sculpteur Sarkis. En 1976, il photographie un graffiti à proximité de l'U-Bunker, dont le cliché constitue un dialogue constant entre l'artiste et le signe peint, aboutissant ainsi à des réalisations variées. La même année, il présente un premier ensemble de peintures associé à de multiples photographies du graffiti anonyme lors de l'exposition Autopsie d'une peinture anonyme murale en face de la Base Sous-marine à Bordeaux dans les salles du jeune C.A.P.C. (Centre d'Arts Plastiques Contemporains) de Bordeaux. Après avoir présenté sa production dans diverses galeries européennes, l'ensemble de son travail plastique lié au graffiti bordelais et également à celui découvert dans la rue Vergniaud à Paris fait l'œuvre d'une rétrospective intitulée Réserves Accessibles au Musée National d'Art Moderne de Paris en 1976. Parmi les peintures présentées, une série de soixante-six bâches peintes entre 1976 et 1978 est destinée à « aller pour toujours dans la base sous-marine allemande ${ }^{8}$ » afin de retrouver leur lieu d'origine. Au mois de mai 1980, Sarkis installe son travail grâce au soutien du C.A.P.C. sur le pourtour d'une des alvéoles de la base sous-marine de Bordeaux dans le cadre de l'exposition Réserves sans retour...9. Cette initiative "sauvage » constitue la première véritable exposition temporaire abritée par la base bordelaise. Il faudra attendre plus de dix-huit ans avant que cela ne se reproduise.

5 À l'aube des années 1980, l'avenir de la base sous-marine est lié à celui du port de Bordeaux dont l'activité décline inexorablement face aux complexes portuaires modernes de Blaye, Bassens, Le Verdon et Ambès, situés en amont de la Garonne (fig. n³). Malgré 
de nouveaux aménagements réalisés sur le pourtour des quais de Bacalan, le secteur des bassins à flot est déclassé par le Port Autonome de Bordeaux le $1^{\mathrm{er}}$ janvier 1982. Suite à cette annonce, les entrées de la base sous-marine sont murées et son accès est strictement interdit au public pour d'évidentes raisons de sécurité. Toutefois, une éventuelle destruction du bunker est rapidement écartée, à la fois par les spécialistes conscients des risques liés à l'instabilité du sol et par les finances de la ville de Bordeaux ${ }^{10}$. Face à cette radicale et onéreuse alternative, les premiers projets de reconversion du secteur des bassins à flot, englobant la base sous-marine, voient le jour.

Figure 3

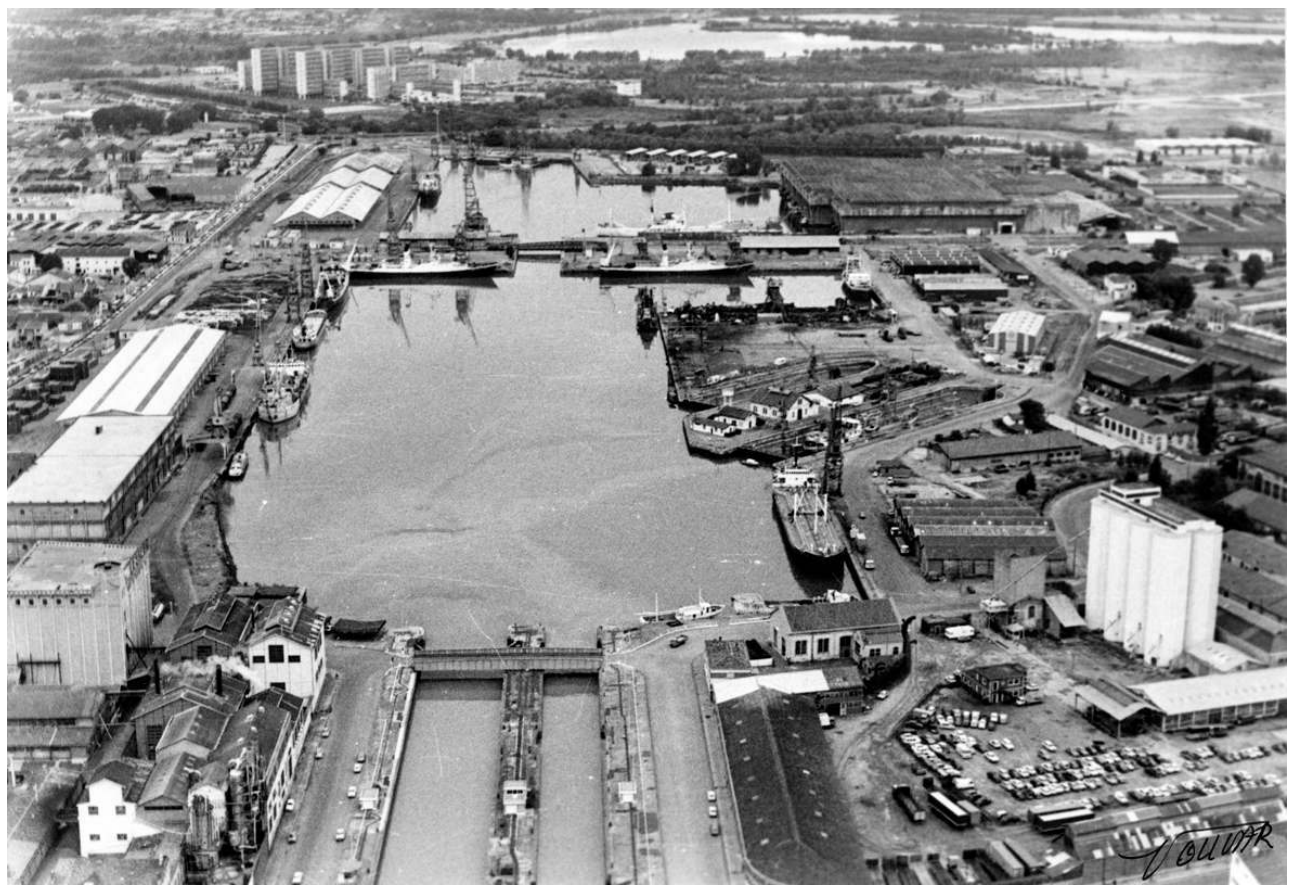

Vue aérienne des bassins à flot en 1975 .

Phot. Vincent Olivar. () La Mémoire de Bordeaux.

6 En 1982, Francis Martin, alors étudiant à l'École Nationale Supérieure d'Architecture et de Paysage de Bordeaux, constate que la situation moribonde de Bacalan, entre friche industrielle et militaire, empêche le développement de la ville vers le nord et que les bassins à flot constitueraient un site idéal pour insuffler une nouvelle vie au quartier. Dans le cadre de ses recherches, l'architecte produit un vaste projet d'aménagement, présenté en 1983 à Jacques Chaban-Delmas ${ }^{11}$. De cette entrevue résulte la formation d'un groupe de travail chargé d'affiner le projet originel et de concevoir l'ébauche d'une «marina urbaine», constituée d'un port de plaisance, d'une cité sur pilotis, d'équipements liés au commerce et au tourisme, de logements et d'ateliers sur les pourtours des bassins à flot. Enfin, la base sous-marine serait transformée en un Écomusée de la mer, les alvéoles permettraient l'accueil de navires en mouillage, alors que sa surface intérieure pourrait servir à des expositions, à l'instar de ce qui a été fait à Londres avec les Docks Sainte-Catherine. L'absence de moyens financiers conséquents, nécessaires à l'aménagement des bassins à flot de Bacalan empêche cependant cet ambitieux projet de voir le jour. À défaut de trouver preneur, la base sous-marine continue d'attiser des velléités architecturales comme celles de Philippe Lamarque et de Denis Lacroix qui en 1988 espèrent y installer un espace dédié à la promotion du vin de 
Bordeaux, Vinopolis. Selon leur projet, la surface intérieure du U-Bunker permettrait le stockage de cuves à vin, le bâtiment d'annexe serait occupé par des bureaux et un restaurant panoramique, le toit servirait d'immense parking, alors que la face nord remblayée de sable pourrait se transformer en dune. Ce projet démesuré, relayé par la presse locale, ne vit jamais le jour ${ }^{12}$.

\section{Un conservatoire pour la base sous-marine}

7 Le début des années 1990 est synonyme de nouvelles orientations pour la base sousmarine avec l'installation du Conservatoire International de la Plaisance de Bordeaux (C.I.P.B.) dans ses murs. L'ouverture au public de cette structure en juillet 1993 est le fruit de la convergence de volontés communes, à savoir celle de ses promoteurs, Daniel Charles et Jean-Bertrand Mothes Massé (respectivement historien du yachting et régatier bordelais) et celle de la municipalité qui cherchait une affectation à la base sous-marine par l'intermédiaire de la Chambre de Commerce et d'Industrie de Bordeaux. En effet, depuis 1989 un groupe de travail étudiait l'avenir de la zone des bassins à flot avec notamment le souhait de constituer un parc dédié au nautisme tout en développant la vocation culturelle et muséographique de la base sous-marine ${ }^{13}$. Le projet de Daniel Charles et Jean-Bertrand Mothes Massé tombait donc à point nommé pour la ville de Bordeaux qui s'est investie dans cette opération d'intérêt général favorisant le développement du quartier "difficile» de Bacalan. La Mairie a même obtenu la responsabilité de la gestion d'une partie de la base sous-marine suite à la signature d'une convention d'occupation pour un franc symbolique versé au Port Autonome de Bordeaux afin de prendre en charge le coût de l'ensemble des travaux de réfection du site. Le projet $\mathrm{du}$ conservatoire bordelais s'articule alors autour de quatre axes: la conservation muséographique, la navigation, la recherche et la formation. Dans l'esprit de son promoteur, Daniel Charles, le C.I.P.B. se devait de combler un manque dans le paysage muséal national et international : "Cette idée est le fruit d'un constat tout simple. Il existe le Musée de l'air et de l'espace au Bourget, le Royal Air Museum de Londres, le San Diego Aerospace Museum aux U.S.A., tous mondialement connus et accueillant des originaux de l'histoire de l'aviation. Et rien pour la navigation de plaisance, de loisirs comme de compétition, alors que cette discipline ancienne et tout aussi riche que l'aviation ${ }^{14}$ ». L'engagement d'investisseurs publics et privés permet à Daniel Charles et Jean-Bertrand Mothes Massé d'établir un projet culturel cohérent avec une visée internationale. De plus, l'absence d'autres structures identiques à l'échelle mondiale permet aux deux hommes de réunir à Bordeaux des pièces de collection disséminées aux quatre coins du monde ${ }^{15}$. C'est ainsi que la base sous-marine abrite les pièces majeures de l'histoire du nautisme avec notamment une reconstitution du plus ancien catamaran européen, le Simon and Jude (1662), les plus vieux canots d'aviron (1860) et voilier de régate (1892), le prototype Ville-de-Paris de Marc Pajot engagé lors de l'America's Cup, l' Hérétique d'Alain Bombard, ou encore le premier voilier à atteindre en 1980 les 36 nœuds : le Crossbow 2. Douze parcours de visites offraient aux visiteurs la possibilité de découvrir plus de soixante-dix bateaux, une centaine de maquettes et une cinquantaine de pièces mécaniques exposées dans les cinq premières cellules et alvéoles du bunker. Les salles de l'annexe étaient notamment investies pour l'occasion, à l'image de l'ancien réfectoire de la base. Cinq mois après son ouverture, le C.I.P.B. a déjà accueilli près de vingt mille visiteurs, davantage attirés par la découverte d'un monument dont l'accès leur était jusqu'alors interdit que par les collections qu'il abrite. De ce fait, la mauvaise gestion 
financière et muséographique de cette structure dans un premier temps associative puis publique par Daniel Charles et Jean-Bertrand Mothes Massé se traduit par l'établissement d'une nouvelle hiérarchie avec la nomination de Jean-Pierre Jamay à la tête du C.I.P.B. au début de l'année 1994. Ainsi, le conservatoire doit avant tout s'implanter au niveau du tourisme régional et trouver sa place dans la politique culturelle de la ville avant d'envisager une possible envergure internationale. Les douze parcours précédemment évoqués sont remplacés par trois approches historiques orientées autour de la navigation : le motonautisme, la plaisance en Aquitaine et les grandes courses nautiques. Le responsable du C.I.P.B. instaure également la mise en place d'activités dédiées à la jeunesse et donne une place plus importante aux vidéos, bornes interactives et expositions photographiques afin d'alléger le contenu des collections, difficilement accessible intellectuellement pour une personne «non initiée». En 1996, le C.I.P.B. clôture sa quatrième saison et malgré de louables efforts, le conservatoire est boudé par le public. Depuis son ouverture, il a tout de même convaincu plus de cent dix mille visiteurs, mais le non-renouvellement des collections et le peu d'intérêt porté par le public bordelais au nautisme poussent Jean-Pierre Jamay à considérer son musée comme «inerte ${ }^{16}$ ». À la fin de l'année 1997, le Conservatoire International de la Plaisance de Bordeaux ferme définitivement ses portes. Pour autant, la base ne perd pas définitivement sa vocation nautique. En effet, une partie des collections du C.I.P.B. continue d'être exposée jusqu'à la fin des années 1990, au travers d'un espace dédié à la plaisance en Aquitaine.

\section{Un nouvel équipement culturel}

\section{Du « métal » à l'art contemporain}

8 À partir de 1996, les bateaux de plaisance du C.I.P.B. ne sont plus les seuls occupants de la base sous-marine. En effet, sous l'impulsion de Jean-François Buisson, sculpteur et batteur dans le groupe de métal bordelais Spina, un studio d'enregistrement est aménagé dans l'alvéole 9 du bunker suite à la signature d'une convention d'occupation précaire entre la mairie de Bordeaux et l'artiste. Les membres de Spina développent alors un espace dédié à l'art total en y introduisant un atelier d'infographie, de répétition, de sculpture ainsi qu'un studio fond bleu pour y réaliser des incrustations vidéos ${ }^{17}$. Alors sous contrat avec deux grandes maisons de disques, Polygram et Musidisc, Spina obtient l'accord du directeur du C.I.P.B. pour la programmation d'un concert à l'intérieur de la base sousmarine, le 27 septembre 1997, jour de sortie de leur album Le meilleur des mondes. Les trois mois de préparation technique nécessaire à la bonne tenue du concert dans la cellule $4 \mathrm{du}$ bunker sont récompensés par une affluence record, plus de mille personnes assistent à l'événement. Des retardataires sont même refusés, le concert affichant complet à une époque ou le C.I.P.B. en pleine restructuration peinait à séduire de nouveaux visiteurs. Si le nautisme est boudé par le public bordelais, la base sous-marine s'avère être un formidable écrin pour les expositions d'art contemporain comme le prouve l'exposition Sous le béton la plage réalisée par l'association Art AttAcks, présidée par Thierry Barrera, en septembre 1998. De ce fait, cette initiative regroupant la production de vingt-six artistes est la première exposition d'art contemporain abritée par la base sous-marine depuis 1980 et les installations sauvages de Sarkis. Peintures, sculptures, installations, 
architecture, vidéo et design se mêlent à l'architecture de la base sous-marine pour une expérimentation annonciatrice.

9 À l'automne 1998, le bunker est fermé au public dans le cadre d'une importante campagne de travaux permettant à la base sous-marine de répondre aux nouvelles exigences du public et à la nouvelle orientation du lieu ${ }^{18}$. Dans un premier temps, l'entrée de la base sous-marine est restructurée, pour cela le mur nord-est (extérieur de l'alvéole 1) est percé afin d'y établir une nouvelle entrée. Une passerelle enjambant les eaux de l'alvéole 1 est installée dans le prolongement de la nouvelle ouverture. Le quai séparant les alvéoles 1 et 2 est aménagé afin de permettre l'accès aux visiteurs. À ces restructurations, il faut ajouter des opérations de rénovations des bureaux présents dans l'annexe, des travaux d'étanchéité, la pose d'alarme ou d'équipements de traitement d'air, la modification du réseau électrique, de la plomberie, des sanitaires ainsi que l'implantation d'une terrasse sur le toit de l'annexe dotée d'un escalier hélicoïdal donnant accès au toit. Un espace de douze mille mètres carrés est aménagé afin d'assurer la mutation d'une architecture militaire en équipement culturel avec notamment la réalisation d'une salle de spectacle modulable d'une capacité de deux cent soixante personnes. Ainsi, une nouvelle base sousmarine ouvre ses portes durant l'été 1999 avec une programmation orientée vers la production artistique contemporaine. Expositions permanentes et temporaires se côtoient dans un espace allant de l'annexe à la quatrième cellule du bunker jusqu'alors non investie par le C.I.P.B. L'exposition Une saison photographique présente alors les travaux de huit photographes se situant en adéquation avec le site, à l'image du projet $U$ Base mené par la photographe Isabelle Kraiser et le plasticien Patrick Marty ${ }^{19}$. Leur intervention commune sur le toit de la base sous-marine constitue une première par sa localisation, mais aussi par la transformation du toit du bunker en support et modèle de leurs réalisations éphémères (fig. $\left.\mathbf{n}^{\circ} \mathbf{4}\right)$. 


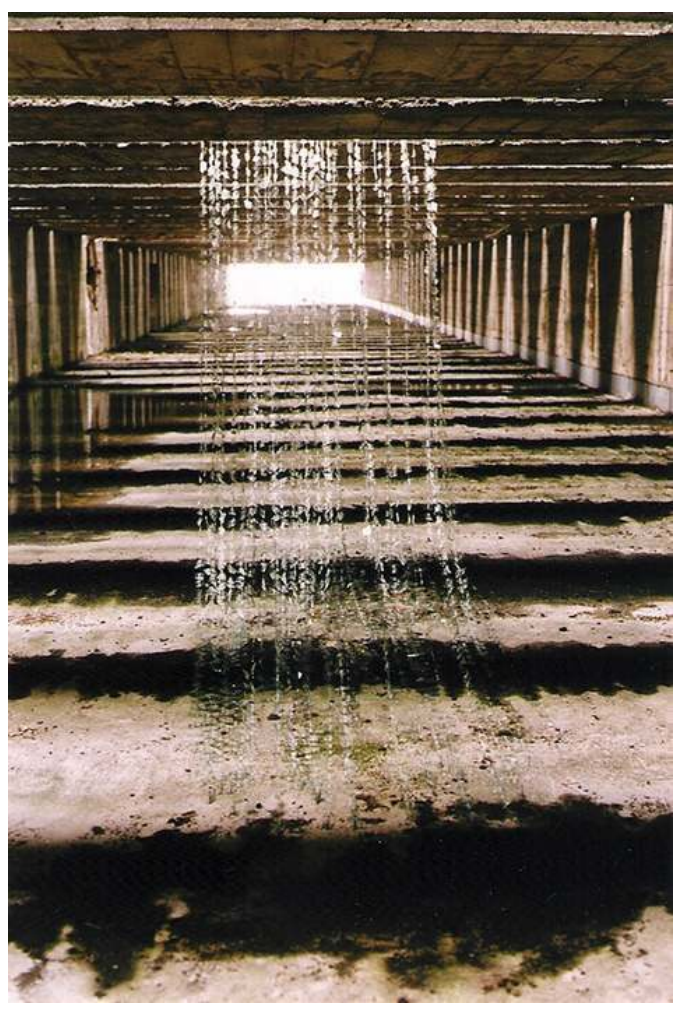

Installation de verres sur des fils en nylon sous les structures Fangrost de la base sous-marine par Patrick Marty, 1999.

(C) Isabelle Kraiser/Patrick Marty.

Enfin, à la même époque, l'association Art AttAcks investit une seconde et dernière fois les entrailles de la base sous-marine avec une exposition intitulée Dans le ventre de la bête. Alors que la base sous-marine gagne en visibilité auprès du public bordelais, la municipalité confie en 2000 le plan de mise en lumière du monument aux architectes Jean de Giacinto et Jean-Hugues Seurat. Plus de deux kilomètres de câble et quatre-vingts spots sont nécessaires à la réalisation de leur étude, à savoir la cohabitation entre des lumières rouges éclairant la coque de béton de la base et des lumières blanches dévoilant la toiture et les ouvertures (fig. $\mathbf{n}^{\circ}$ ). La volonté de mettre en lumière l'architecture des bases sousmarines françaises n'est pas un phénomène exclusif à la ville de Bordeaux. En effet, dès 1991, l'artiste Yann Kersalé avait habillé de lumière la base sous-marine de Saint-Nazaire dans le cadre de la "Nuit des Docks ", initiative encore d'actualité ${ }^{20}$. En 1997, le photographe Michel Semeniako avait investi de nuit la base Kéroman 3 à Lorient en transformant, à l'aide de projecteurs colorés portables, les immenses portes blindées et murs bétonnés du bunker ${ }^{21}$. De ce fait, l'intervention d'artistes auprès des bases sousmarines, et plus spécifiquement celles des villes de Bordeaux, Lorient et Saint-Nazaire, entraîne un regain d'intérêt de la part des populations locales auprès de ce type d'architecture difficilement appréhendable. L'introduction d'activités culturelles, et plus spécifiquement artistiques, se révèle être le principal vecteur stimulant la renaissance des U-Bunker. C'est en tout cas vers cette voie que se tourne la base sous-marine de Bordeaux au début des années 2000 . 
Figure 5

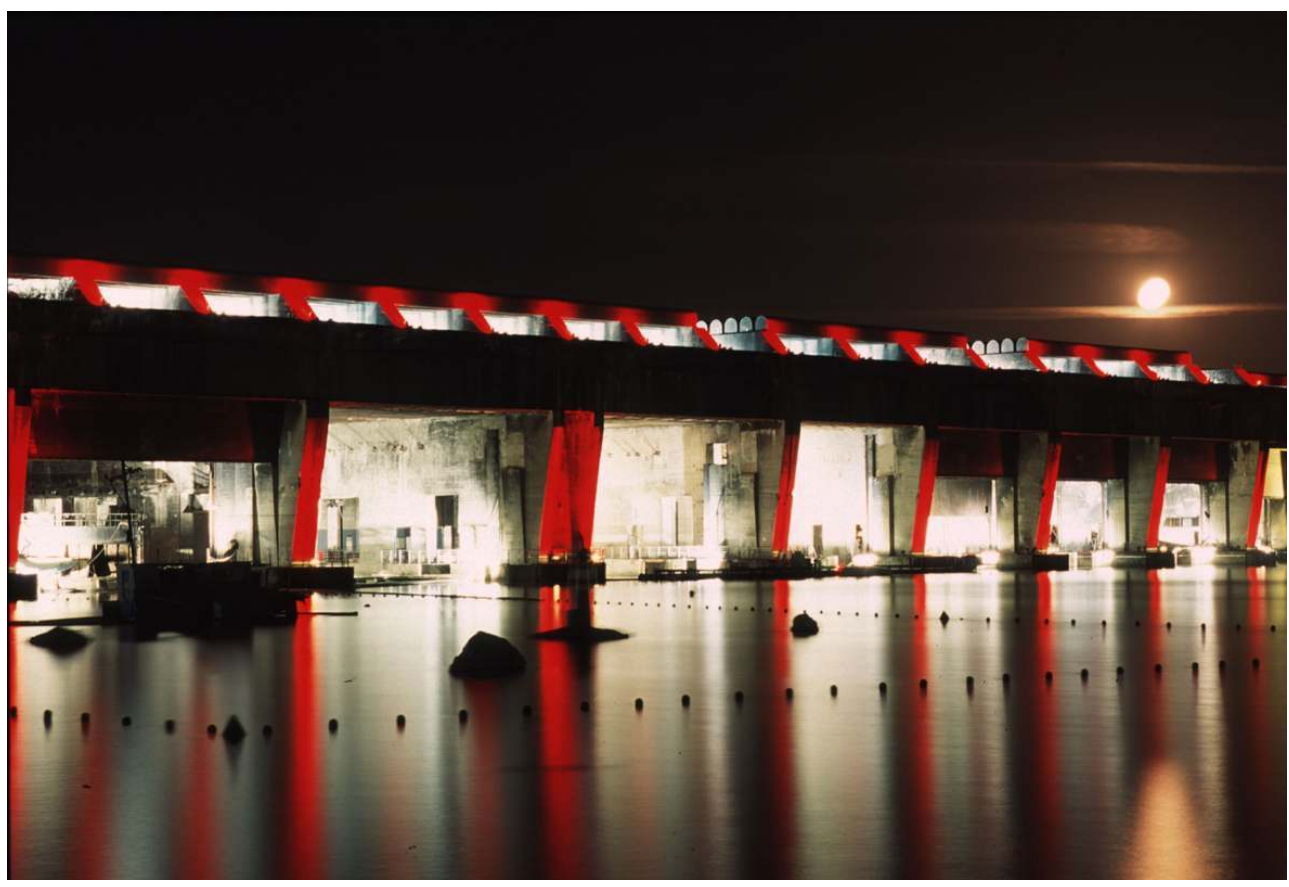

Vue nocturne du dispositif d'éclairage mis en place par Jean Giacinto et Jean-Hughes Seurat en 2000. (c) Thomas Sanson/Mairie de Bordeaux.

\section{Une diversité plébiscitée}

11 La nomination de Danièle Martinez en septembre 2000 à la tête de la direction de la base sous-marine permet à cet imposant équipement culturel de trouver définitivement sa vocation. Alors que Jean-Pierre Jamay avait commencé à ouvrir la base aux spectacles vivants et aux expositions temporaires, tout en conservant les collections héritées du C.I.P.B., l'ancienne organisatrice du Salon du Livre de Bordeaux ne croit pas à la vocation nautique de la base. Elle souhaite « exploiter la particularité de ce lieu, la mettre en scène pour mieux valoriser ce qu'on y met à l'intérieur ${ }^{22} »$. Suite à une réflexion sur le potentiel et les contraintes du bunker, la directrice de la base sous-marine considère que le meilleur moyen pour que la base prenne sa revanche sur l'histoire est d'assurer le retour de l'humain au cœur des dédales du bunker. Qui mieux que les artistes peuvent apporter une telle vocation? Cependant, les œuvres présentées doivent entrer en résonance avec le gigantisme de la base, proposer un défi aux spectateurs ou se situer en adéquation totale avec l'esprit du bunker tout en conservant son identité de friche militaire. C'est avant tout la naissance d'émotion par la contemplation des œuvres qui est attendue dans un espace inédit dans le but de réconcilier les visiteurs avec la base sous-marine. Les expositions n'ont pas de prétention éducative, hormis celle de sensibiliser le grand public à l'art. La conception d'expositions lisibles et intellectuellement accessibles pour des visiteurs non initiés à l'art contemporain assure à la base sous-marine une fréquentation annuelle stable, de plus la gratuité des expositions rompt avec l'accès payant proposé par le C.I.P.B. et ce, dans l'optique d'attirer la population locale et les touristes internationaux. Parmi les cinquante et une expositions temporaires programmées entre 2000 et 2011 à la base sous-marine de Bordeaux, trois tendances muséographiques se 
dégagent, soit la promotion des artistes bordelais et des initiatives locales, les monographies d'artistes internationaux et des grandes figures de l'histoire de l'art, ainsi que le cycle annuel Des Photographes pour l'Histoire.

Il n'est jamais évident pour un artiste d'exposer dans un lieu où sa production doit faire face à de nombreuses contraintes matérielles ou physiques. Nombreux sont les artistes dont le travail est incompatible avec la base sous-marine de Bordeaux, malgré le grand intérêt de leur production. L'espace est complexe, il contraint les œuvres à tisser un lien avec lui, sans cela aucune exposition ne peut exister dans la base sous-marine. Ce procédé de confrontation aboutit à une nouvelle lecture de l'œuvre au travers du lieu, ce dernier lui donnant un autre sens, le plus souvent dramatisé. Ainsi, les artistes bordelais sont les premiers à bénéficier de la reconversion du C.I.P.B. en un espace d'exposition tout en se confrontant aux salles sombres du bunker. Considéré comme un « endroit (...) archiprimal et monolithe ${ }^{23}$ » lors de l'exposition Présence, Regards en 2006 par le peintre Serge Labégorre, l'espace d'exposition de l'annexe de la base sous-marine est capable d'accueillir une centaine de portraits en grands formats mis en valeur par l'éclairage directionnel des salles ainsi que par un parcours scénographique déroutant sur fond de musique classique. En 2009, le peintre Alain Bergeon et le sculpteur Robert Kéramsi présentent à la base sous-marine l'exposition commune À corps découverts ${ }^{24}$.

Tous deux réalisent des œuvres intimement liées au corps, son image, sa gestuelle, ses émotions et ses sentiments. De ce fait, les réalisations colorées et pleines de vie d'Alain Bergeon correspondent avec les sculptures de corps mis à nu de Robert Kéramsi dans un dédale de salles impressionnantes pour les visiteurs qui passent, au détour d'un couloir, d'une pièce basse de plafond à une véritable cathédrale troglodyte coulée dans le béton (fig. $\mathbf{n}^{\circ}$ ). Outre la peinture et la sculpture, la photographie tient aussi une place importante dans la programmation de la base sous-marine. À sept reprises, l'association Itinéraires des Photographes Voyageurs a investi le bunker bordelais avec des productions uniquement en noir et blanc, chaque édition étant caractérisée par la diversité des profils des participants. Photographes reconnus et jeunes artistes s'y côtoient autour de confrontations axées sur le regard à l'image de Stéphane Klein, Jean-Baptiste Huynh, Bernard Plossu, Marcelo Fuentes ou Klavdij Sluban. De plus, la base sous-marine accueille des initiatives culturelles locales telles que Novart, Evento, Les Grandes Traversées, le Festival du Court, les Marches de l'Été..., le temps d'une journée ou d'une soirée pour des représentations de théâtre, des projections cinématographiques, des spectacles de danse ou des concerts. 


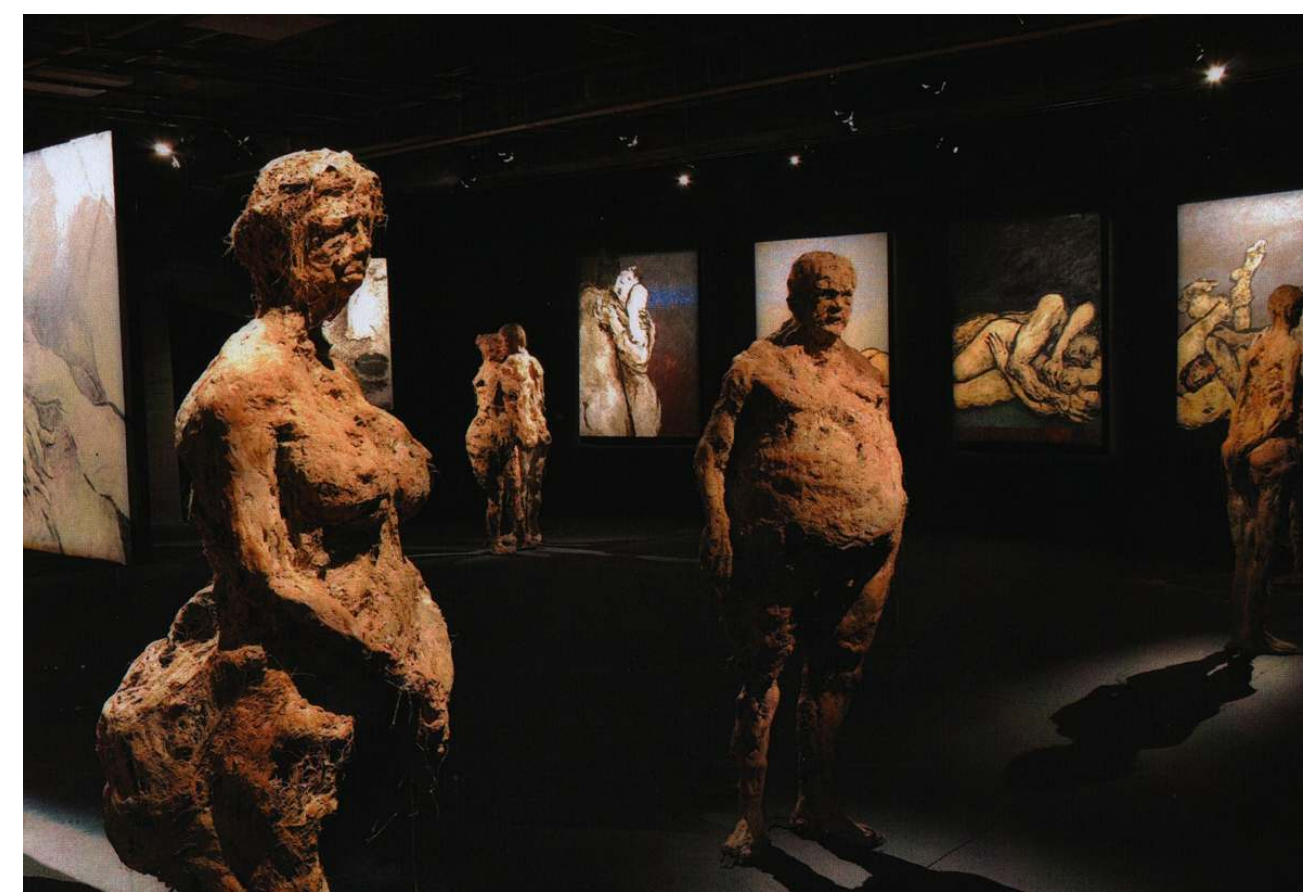

Scénographie de l'exposition À corps découverts présentée à la base sous-marine du 5 mai au 5 juillet 2009 avec les sculptures de Robert Kéramsi et les peintures d'Alain Bergeon.

(c) Alain Bergeon/Robert Kéramsi.

14 L'espace intérieur de la base sous-marine présente de nombreuses contraintes techniques, à l'image du système d'éclairage, la lutte incessante contre l'humidité, la sécurité ainsi que l'échelle des pièces. La modularité des salles de l'annexe, bien que cloisonnées par plusieurs tonnes de béton, permet d'occuper un espace prédéfini selon le format et le nombre d'œuvres à présenter. De plus, un important réseau de couloirs permet d'offrir aux visiteurs un parcours différent pour chaque exposition. Cependant, dans le cadre de la présentation d'expositions d'envergure comme 6 milliards d'Autres en 2010, la base sous-marine retrouve la configuration héritée du C.I.P.B., à savoir l'occupation de l'annexe et des quatre premières cellules. Les vingt et un films d'une durée totale de plus de onze heures, réalisés par Yann Arthus Bertrand, sont notamment projetés sur écran géant dans onze containers rompant ainsi avec la verticalité du lieu et sur écran géant installé dans la cellule $1^{25}$ (fig. $\mathbf{n}^{\circ} 7$ ). À l'image de cet événement, la base sous-marine accueille de grandes expositions monographiques présentant le travail d'artistes de renommée internationale. En 2009, le cinéaste israélien Amos Gitaï est invité par Didier Faustino à investir les murs de la base sous-marine lors d'Evento, festival d'art contemporain de la ville de Bordeaux, en y présentant des extraits de documentaires et de fictions qu'il a réalisés. Inviter un artiste d'origine juive dans ce monument emblématique, cicatrice de l'occupation militaire de la ville et de la folie d'un homme, attribue une charge symbolique très forte à l'exposition. Malgré l'épaisseur du béton, la cacophonie produite par la lecture simultanée d'une quinzaine d'extraits de la production d'Amos Gitaï sur les murs internes de la base dégage une ambiance lourde et bruyante guidant le visiteur au travers d'une profusion d'images qui ne laisse pas la place à une quelconque indifférence à l'instar d'une scène issue du long-métrage Free Zone où l'actrice 
américano-israélienne Natalie Portman pleure pendant dix minutes en écoutant des champs israéliens.

Figure 7

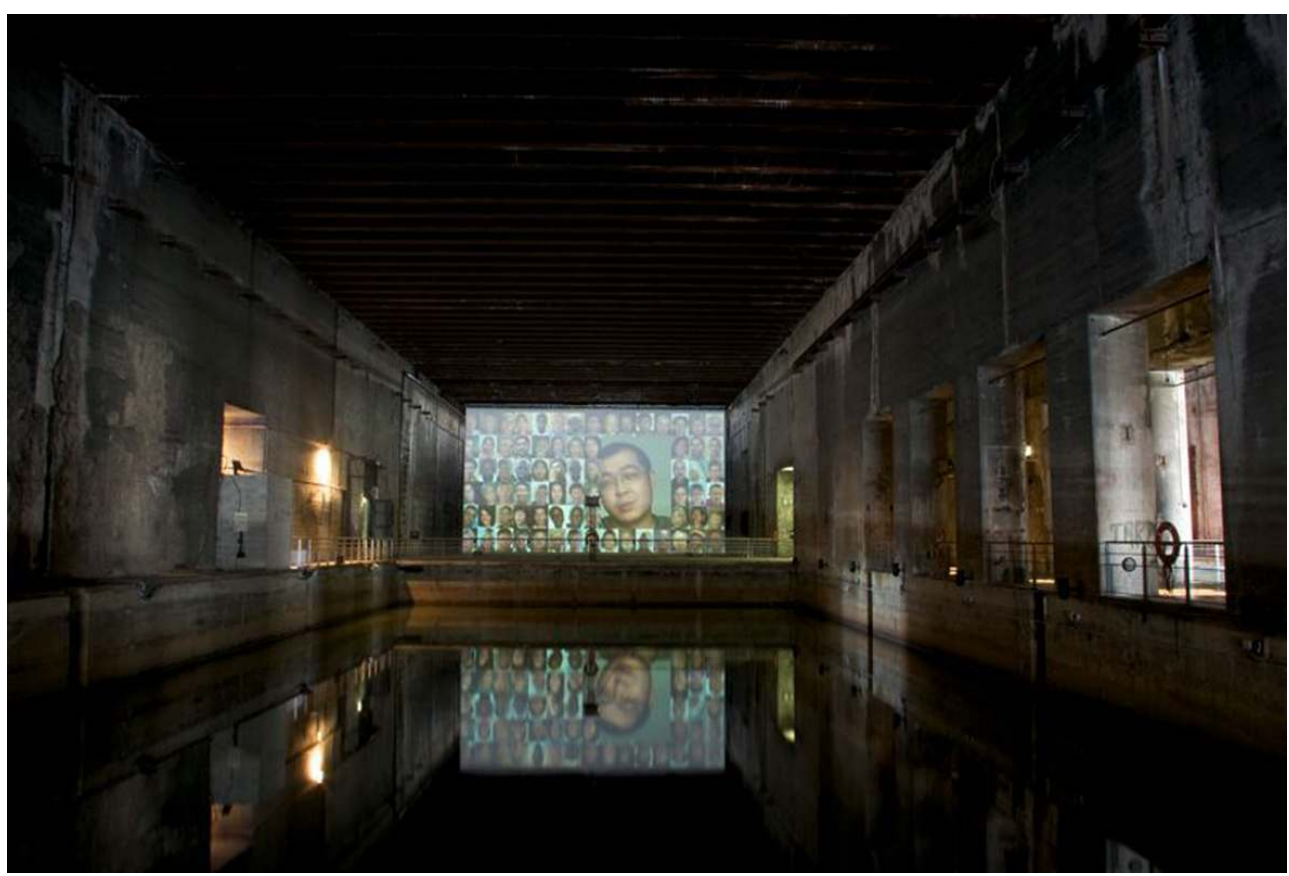

Mosaïque de visages projetée sur un écran de $20 \mathrm{~m} \times 11 \mathrm{~m}$ dans la première cellule de la base sousmarine durant l'exposition 6 milliards d'Autres (9 juillet - 26 septembre 2010).

(c) 7 milliards d'Autres/Fondation GoodPlanet.

15 Ce type de confrontation se révèle être essentiel, à la fois entre les œuvres et les visiteurs mais aussi entre différents artistes intervenants dans la base sous-marine. C'est ainsi que les réalisations de Pierre Alexandre et de Ferrante Ferranti sont présentées en commun autour des gravures de Giovanni Battista Piranesi dans le cadre de l'exposition Imaginaires des ruines en 2008. Le parcours de visite est pensé comme « une confrontation imaginaire entre les estampes du graveur, les photographies de Ferrante Ferranti et les sculptures de Patrice Alexandre ${ }^{26}$ » structuré par des jeux d'ombres et de lumières. La base sous-marine s'affirme comme un lieu idéal pour la réception d'une telle exposition, son architecture porte les marques de son passé, ses murs sont rongés par le temps, la nature y reprenant ses droits par endroits au cœur d'une esthétique typiquement piranésienne. Les ressemblances troublantes entre les productions des trois artistes issus d'époques et milieux différents expriment la fragilité de nos civilisations et questionnent sur la nature de l'héritage des générations futures.

Le lien majeur existant entre les expositions et la dimension historique est également perceptible dans la volonté de Danièle Martinez pour « s'adapter au passé du lieu ${ }^{27}$ » en programmant le cycle d'expositions Des Photographes pour l'Histoire à partir de 2004. La confrontation d'images témoin datant des années précédant et suivant la Seconde Guerre mondiale avec le bunker débute avec Des Européennes et les clichés d'Henri Cartier Bresson. L'année suivante, Robert Capa, un de ses amis photographes avec lequel il a fondé l'agence coopérative Magnum Photos, en 1947, voit sa production présentée dans les salles de la base. Les visages de l'Histoire permettent de présenter plusieurs dizaines de clichés réalisés par l'unique photographe présent lors du débarquement allié en 
Normandie. En 2006, Robert Doisneau et Willy Ronis portent un regard croisé sur la période de l'Occupation, des rues parisiennes désertes à l'heure de la parade allemande à la joie des prisonniers de guerre de retour en France au sein de leurs expositions respectives 40 / 44 et Le printemps refleurira. Lors des éditions de 2007 et 2008, les années de guerre sont pour un temps oubliées afin de laisser place à l'Europe de l'Est face aux prémices de la Guerre Froide avec les travaux d'Erich Lessing à Budapest et les épisodes symboliques de Mai 68 à Paris et à Toulouse vus par Bruno Barbey, Gilles Caron et Jean Dieuzaide. L'édition 2010 met en valeur un autre membre fondateur de l'agence Magnum Photos en la personne de George Rodger avec l'exposition Sur la route 1940-1949, les photographies de Louis Stettner sont présentées en 2009 lors de l'exposition Paris // New York. Le parcours est constitué de scènes de rue, de l'adversité new-yorkaise aux déclarations d'amour parisiennes mettant à rude épreuve la sensibilité des spectateurs. Les travaux de Louis Stettner traduisent son désir de mettre l'humain au centre de ses préoccupations, de créer des liens sociaux entre les hommes, cette volonté trouve un écho favorable dans la démarche de Danièle Martinez qui souhaite réintroduire «l'humain » dans les murs de la base. L'essentiel des expositions présentées dans le cadre Des Photographes pour l'Histoire est réalisé par l'intermédiaire des collections de l'agence Magnum Photos. De ce fait, ce cycle fait de la base sous-marine un lieu incontournable pour les rétrospectives de grands photographes humanistes.

Malgré son relatif éloignement du centre-ville de Bordeaux et ses voies d'accès mal signalées, la base sous-marine accueille en moyenne quarante mille visiteurs par an, constitués de bordelais dont l'attrait pour l'architecture de la base est à chaque fois renouvelé par la programmation d'expositions inédites, sans oublier l'afflux de touristes étrangers qui découvrent les espaces de cet équipement culturel le temps d'une exposition. En vingt ans, les directeurs successifs de la base sous-marine ont su sortir ce lieu de l'oubli, offrir la possibilité à des artistes de s'y produire et de populariser le lieu en y présentant des expositions inédites, stimulant au passage la mutation du quartier voisin des bassins à flot.

\section{La base sous-marine, un patrimoine entre friche militaire et industrielle}

\section{Un patrimoine oublié ?}

Depuis le déclassement des bassins à flot de Bacalan par le Port Autonome de Bordeaux en 1982, les cent quatre-vingts hectares de terrains bordant les bassins et la base sont considérés comme des friches industrielles et militaires. Ce type d'espace attise un désir de projection de la part des municipalités mais aussi des artistes : si le cas de la friche de la Belle de Mai à Marseille constitue un bel exemple de réhabilitation culturelle, beaucoup d'espaces restent encore sous-exploités ${ }^{28}$. C'est le cas des bassins à flot de Bacalan où le mode d'occupation de la base sous-marine répond tout d'abord à un phénomène marginal qui s'amplifie au début des années 1970, époque où les friches de différentes natures deviennent des alternatives aux institutions culturelles placées sous la tutelle des pouvoirs publics. Alors que des bunkers de petite taille sont détruits par certaines municipalités dans le but de requalifier des espaces maritimes ou dunaires, les bases sousmarines s'imposent dans le paysage français comme un patrimoine "par défaut ». En effet, dans chacun des ports français occupés par des U-Bunker, la tentation a été grande 
à la Libération de vouloir détruire ces vestiges gênants et encombrants au regard de l'Histoire. Cependant, l'importance des coûts provoqués par un tel chantier est inabordable pour n'importe quelle ville française à l'heure où l'urgence est à la reconstruction. L'analyse de l'évolution de l'intérêt porté par le public auprès des vestiges du Mur de l'Atlantique produite par Christelle Neveux permet de mettre en lumière la manière dont les blockhaus sont devenus des arguments touristiques au début des années $1990^{29}$. Notamment avec l'éclosion d'espaces culturels constitués dans les bases sous-marines, à l'image de celui de Bordeaux, mais également des petits musées établis dans des postes de défenses du Mur de l'Atlantique, comme Le Musée de la Guerre à Calais ou le Mémorial de la bataille de l'Atlantique à Camaret. Néanmoins, les bases sousmarines du Mur de l'Atlantique bénéficient actuellement d'une faible reconnaissance patrimoniale. Seule celle de Saint-Nazaire jouit du label «Patrimoine du XX $\mathrm{XX}^{\mathrm{e}}$ siècle » depuis 2010 alors que le slipway du port de La Rochelle, situé dans le dispositif de la base sous-marine de la ville, bénéficie également du label « Patrimoine du $\mathrm{XX}^{\mathrm{e}}$ siècle » et d'un classement au titre des Monuments Historiques depuis 200230. À Bordeaux, les aboutissements du processus de patrimonialisation de la base sous-marine sont de natures différentes. D'un point de vue humain, la patrimonialisation prend forme au début des années 1990 avec l'installation du C.I.P.B. dans le monument. L'identité militaire du lieu est remise en question au profit d'un aspect inédit, celui de la plaisance et de la culture. Ici, ce processus relève essentiellement de la politique culturelle de la ville aboutissant indirectement à une opération d'intellectualisation, comme l'a décrit Aloïs Riegl: «ce n'est pas leur destination originelle qui confère à ces œuvres la signification de monument, c'est nous, sujets modernes, qui la leur donnons ${ }^{31}$ ». De plus, l'introduction de la base sous-marine dans le programme des Journées Européennes du Patrimoine confirme l'intérêt porté par les bordelais à cette architecture. L'émergence d'une telle mobilisation permet la construction de l'élément patrimonial afin de répondre à un certain nombre d'enjeux, dont l'appropriation du lieu par le public. D'un point de vue institutionnel, la base sous-marine de Bordeaux ne bénéficie pas de protection au titre des Monuments Historiques ni du label Patrimoine du XXe siècle. Enfin, malgré sa situation dans le périmètre de «Bordeaux Port de la Lune » inscrit à la liste du Patrimoine Mondial de l'UNESCO depuis 2007, elle ne fait pas l'objet d'un programme de médiation ou d'interprétation ${ }^{32}$.

\section{Une reconversion programmée}

La base sous-marine telle que nous la connaissons aujourd'hui doit être considérée comme une expérimentation culturelle. Elle est le fruit d'une reconversion partielle, le bunker ne comprenant pas de réel espace rénové, seule l'annexe étant équipée afin d'assurer l'accueil d'expositions. Les quatre premières cellules sont utilisées lors d'événements d'envergures, de spectacle d'arts vivants et de concerts, mais les sept autres cellules ne sont pas reconverties. La Mairie assure uniquement la coûteuse mission d'entretien du lieu. Dans l'optique de trouver une fonction à la totalité du bunker et pour dynamiser le secteur des bassins à flot, différents projets d'aménagement ont été confiés par la Communauté Urbaine de Bordeaux, le Port Autonome de Bordeaux et la ville de Bordeaux, aux agences d'architecture et d'urbanisme d'Antoine Grumbach en 1998 et de Nicolas Michelin en 2010. 
En succédant à Jacques Chaban-Delmas en 1995, Alain Juppé présente l'année suivante un projet urbain visant à rendre à la ville sa centralité perdue, à assurer le développement vers la Garonne et à reconquérir le patrimoine. Cette reconquête de l'espace public se joue également dans la zone nord de Bordeaux avec la programmation du désenclavement de Bacalan et la mise en valeur du site des bassins à flot accompagnée d'une redéfinition des activités de la base sous-marine. Il est prévu d'y constituer un espace destiné à la mémoire et à l'histoire maritime de la ville dans les parties non occupées par le C.I.P.B., alors que les bassins à flot doivent devenir « le premier maillon d'un urbanisme culturel ${ }^{33}$ " par la valorisation du patrimoine portuaire. En 1998, l'architecte-urbaniste Antoine Grumbach remporte le concours lancé par la Communauté urbaine et la ville de Bordeaux dans le but d'établir aux bassins à flot une zone de transition entre le centre-ville et Bordeaux-Lac ${ }^{34}$. Antoine Grumbach constate que le problème posé par les bassins bordelais est un phénomène récurrent dans les grandes villes, où des zones industrielles deviennent rapidement obsolètes et délaissées à un moment donné de leur histoire. À Bordeaux, deux quartiers se sont développés en tournant le dos aux bassins à flot, Bacalan et les Chartrons. Après avoir été le poumon de l'économie de la ville, ils doivent s'imposer comme un lieu de carrefour. Le plan guide du secteur des bassins à flot offre la possibilité d'aménager un espace gigantesque (fig. $\left.\mathbf{n}^{\circ} \mathbf{8}\right)$, plus grand que la place des Quinconces ou que le centre historique de la ville. Les aménagements prévus visent à mettre en valeur la base sous-marine considérée par Antoine Grumbach comme « un des éléments importants du quartier, de son histoire, de sa mémoire ${ }^{35}$ ». L'architecte estime que son projet de Zone d'Aménagement Concerté peut être uniquement viable sur le long terme, à partir de l'horizon 2030. Le coût d'aménagements du secteur est estimé à plus de vingt-huit millions d'euros, mais face à une mauvaise conjoncture économique et politique, le projet est définitivement entériné en 2004.

Figure 8

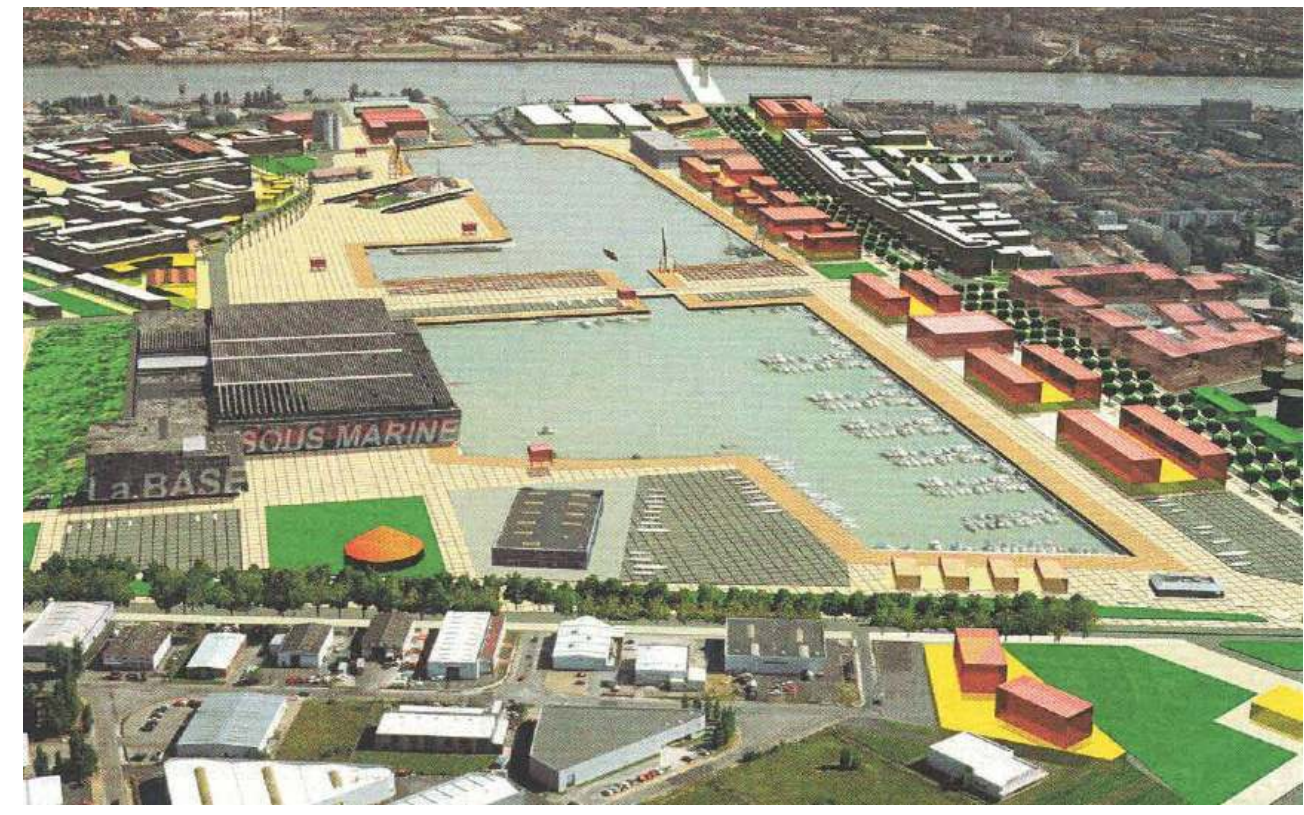

Vue volumétrique des bassins à flot réalisée dans le cadre d'une Zone d'Aménagement Concerté, par le cabinet d'architecture d'Antoine Grumbach.

(c) Antoine Grumbach \& Associés. 
En 2007, la Communauté Urbaine de Bordeaux affiche de nouvelles orientations vers le secteur des bassins à flot et la base sous-marine en annonçant la mise en place d'un Plan d'Aménagement d'Ensemble. La consultation d'urbanistes aboutit deux ans plus tard à la validation du projet proposé par l'agence A.N.M.A. de Nicolas Michelin, déjà en charge des travaux de l'îlot Armagnac dans le quartier Belcier à Bordeaux. Le plan guide proposé traduit la volonté de révéler « le génie ${ }^{36}$ » des bassins à flot aux bordelais (fig. $\mathbf{n}^{\circ} \mathbf{9}$ ), l'architecte considère que ces derniers sont dans « une situation exceptionnelle au débouché des quais dont on voudrait qu'ils se retournent et se prolongent vers les bassins à flot ${ }^{37}$ ». Les douze hectares présents autour des bassins doivent être réhabilités et rendus accessibles au public bordelais par l'installation d'une promenade jalonnée de places dans la continuité du parcours des quais de la Garonne.

Figure 9

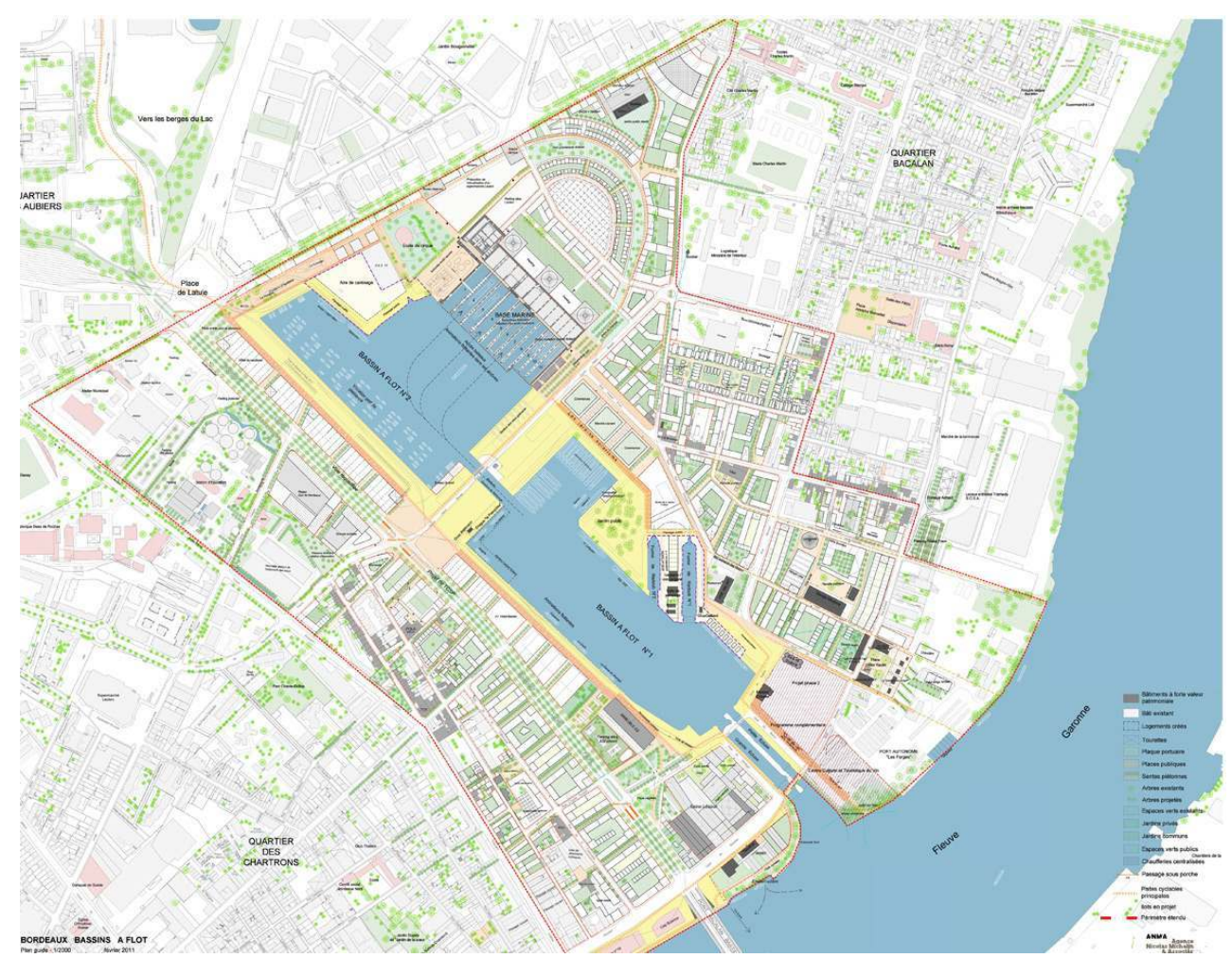

Plan guide d'aménagement, quartier des Bassins à flot.

(c) ANMA Agence Nicolas Michelin \& Associés.

La vie culturelle du quartier s'oriente autour de deux grands pôles situés de part et d'autre des bassins à flot et chargés de renforcer l'attractivité du site, le futur centre culturel et touristique du vin (C.C.T.V.) et l'actuelle base sous-marine. Confié à l'agence d'architecture X-TU, le C.C.T.V. devrait ouvrir ses portes en 2014 sur le site des Forges en bordure de la Garonne à proximité de l'emplacement de l'ancienne écluse couverte. Dans le plan guide de l'agence A.N.M.A., le bunker, renommé «Base Marine», y est décrit comme un « lieu poétique à programmation flottante ». Il est prévu d'établir un passage public traversant la totalité du bunker afin d'accentuer son attractivité en situant son entrée principale au sud, face aux quais. L'aménagement interne traduit deux orientations caractérisées par une intervention minimale tout en assurant la mise en sécurité par des purges des bétons et une inévitable intervention sanitaire. La première 
orientation vise à investir les six premières alvéoles afin de les dédier aux animations flottantes, caractérisées par la présence de bateaux et barges flottantes définitives ou temporaires, destinés à d'éventuels artistes en résidence. La seconde orientation consiste à aménager dans les alvéoles 7 à 11 un cinéma multiplexe flottant évoqué dans un premier temps puis finalement écarté. En effet, la réflexion sur le devenir de la base sousmarine ne doit pas être déconnecté de celle du quartier, destiné à accueillir sur quarantequatre hectares cinq mille cinq cents logements en périphérie des bassins à flot, dont les équipements doivent répondre aux besoins des futurs habitants. À l'arrière du bunker, un parking à trois niveaux d'une capacité totale de neuf cents places doit voir le jour afin d'assurer l'accueil des visiteurs. Enfin, le toit de la base doit être occupé par de la végétation dans le but de préserver le microcosme installé sous les structures Fangrost depuis la fin de la Seconde Guerre mondiale. La question de l'utilisation de l'annexe en temps qu'espace d'exposition n'est pas évoquée dans le plan guide de Nicolas Michelin, il semble néanmoins que la mairie souhaite conserver l'équipement culturel déjà en place.

Si le projet de l'agence A.N.M.A. s'annonce prometteur, il demeure avant tout à l'état de projet, les questions de faisabilité financière et technique restant encore en suspens. Par ailleurs, le développement du quartier résidentiel des bassins à flot et du C.C.T.V. est aujourd'hui prioritaire pour des raisons économiques. Les mots publiés par Michèle Larüe-Charlus, directrice générale de l'aménagement de la ville de Bordeaux dans 2030, vers le Grand Bordeaux, une métropole durable ont le mérite de résumer la situation et d'attiser de futures velléités architecturales sur l'avenir de la base sous-marine: "le projet de la base, soyons clair, nous ne l'avons pas encore ${ }^{38} »$. Et si les cinquante ans d'initiatives artistiques abritées par le bunker ne constituaient pas déjà un programme de reconversion en soi?

\section{NOTES}

1. - Cet article synthétise les recherches menées lors de l'année universitaire 2010/2011 dans le cadre de la rédaction d'un mémoire de première année de Recherche en Histoire de l'art: MARSAN, Mathieu. La base sous-marine de Bordeaux: une expérimentation culturelle. Mémoire de Master I Recherche en Histoire de l'art et patrimoine, sous la direction de Marc Saboya. Pessac: Université Michel de Montaigne, Bordeaux 3, 2011.

2. - «Betasom » est un nom de code composé de l'abréviation Beta pour la lettre de B de Bordeaux et som pour sommergibili, sous-marin en italien.

3. - SALLABERY, Francis. Aquitaine Allemande. Biarritz : J. et D. éditions, 1995, p. 91.

4. - MARZAGALLI, Silvia. Bordeaux et la marine de guerre XVII ${ }^{e}-X^{e}$ siècles. Pessac: Presses Universitaires de Bordeaux, 2002, p. 183.

5. - Voir : DURAND, Claude. Le Coup de grâce : Scénario, dialogues et réalisation de Jean Cayrol et Claude Durand. Paris : Éditions du Seuil, 1965.

6. - Propos rapportés dans : GODFREY, Dominique. « Une base de culture ». Le Festin, mars 2008, $\mathrm{n}^{\circ} 65$, p. 50.

7. - SIGMA. Sigma, 25 ans pour une culture visionnaire à Bordeaux : l'aventure d'un festival. Bordeaux : Sigma, 1990, p. 70. 
8. - Sarkis: « réserves sans retour... ». (Bordeaux, Base sous-marine, exposition permanente organisée par le CAPC en 1980). Bordeaux : Entrepôt Lainé, 1980, p. 6.

9. - On consultera le site Internet de l'artiste afin de découvrir son travail dans la base sousmarine lors de l'exposition Réserves sans retours...: http://www.sarkis.fr/fr/expographievisuels/198089.

10. - VIDALENC, Pierre Laurent. La base sous-marine de Bordeaux, lieu de mémoire, lieu d'avenir. Certificat International d'Écologie Humaine, Talence : Université des Sciences et Technologies au service de l'Homme et de l'Environnement, Bordeaux 1, 1998, p. 72. L'auteur estime un coût de vingt-sept millions d'euros sur trois ans pour le chantier de destruction de la base sous-marine de Bordeaux.

11. - Voir: MARTIN, Francis. Marina urbaine, aménagement des bassins à flot à Bordeaux. T.P.F.E., Talence : École Nationale Supérieure d'Architecture et de Paysage de Bordeaux, 1982.

12. - DEROUDILLE, Jean-Pierre. «Vinopolis émerge à la base sous-marine ». Sud-Ouest, 27 mai 1988.

13. - Voir : KLAUTH, Sophie. La difficile genèse d'un équipement public culturel, rapport de stage réalisé auprès du Conservatoire International de la Plaisance de Bordeaux. D.E.S.S. de Management des Organisations et Entreprises de Service Public. Pessac: Institut d'Études Politiques de Bordeaux, 1994.

14. - Propos rapportés dans : 期Anonyme區. « Le conservatoire international de la plaisance largue les amarres ». Mensuel de la ville de Bordeaux, avril 1993, n² 241, p. 40.

15. - France 3 Aquitaine. «Le conservatoire de plaisance». 22 mai 1993, Ina.fr.: http:// www.ina.fr/art-et-culture/musees-et-expositions/video/RXC08028733/le-conservatoire-deplaisance.fr.html.

16. - GODFREY, Dominique. « Une base de culture ». Le Festin, mars 2008, n 65, p. 50.

17. - FOUQUET, Denis. Bordeaux rock(s). Bordeaux : Le Castor Astral, 2007, p. 369.

18. - Projet pilote urbain "Bordeaux les deux rives" 1998-2000. Archives municipales de la ville de Bordeaux.

19. - RETHORE, Gilles Christian. « Land Art sur le toit ». Sud-Ouest, 6 juillet 1999.

20. - DOSSAL, Philippe, GRAVELAINE, Frédérique (de), MASBOUNGI, Ariella. Saint-Nazaire, ville port . 医s.1医 : Place Publique, 2010, p. 27.

21. - OLLIER, Brigitte. "Lumières sur Lorient, La base sous marine de Keroman ». Géo, janvier $1998, n^{\circ} 227$, p. 34.

22. - Propos rapportés dans : VIDEAU, Noémie. «La base en mutation ». Sud-Ouest, 12 octobre 2001.

23. - 四Anonyme區. «Chronique d'un bombardement de regards». Bordeaux Magazine, juin 2005, n -333, p. 25.

24. - Voir : À Corps Découverts. Bergeon+Kéramsi. (Bordeaux, Base sous-marine, 7 mai-5 juillet 2009). Montreuil : A éditions, 2009.

25. - Voir : 6 milliards d'Autres. Paris, Grand Palais, 10 janvier-12 février 2009. Paris : La Martinière, 2009. On consultera aussi le site de la Fondation GoodPlanet : http://www.7billionothers.org/fr/ node/25.

26. - Voir : Imaginaire des ruines : hommage à Piranèse. Bordeaux, Base sous-marine, 27 mai-31 août 2008 ; Villeneuve-sur-Lot, Musée de Gajac, 30 janvier-19 avril 2009. Arles : Actes Sud, 2009.

27. - GODFREY, Dominique. «Une base de culture ». Le Festin, mars 2008, n 65, p. 52.

28. - Friches industrielles, lieux culturels. Actes du colloque, Strasbourg, 18-19 mai 1993. Strasbourg: La Laiterie, 1994, p. 17.

29. - Voir: NEVEUX, Christelle. Le Mur de l'Atlantique: Vers une valorisation patrimoniale? Paris : L'Harmattan, 2006.

30. - Voir notice de la base Mérimée du Slipway de La Rochelle : PA17000051. 
31. - RIEGL, Aloïs. Le culte moderne des monuments. Paris : Éditions du Seuil, 1984 (1 ère édit. française), p. 43.

32. - Malgré sa situation dans le secteur inscrit à la liste du patrimoine mondial de l'UNESCO, la base sous-marine ne figure pas dans la présentation du site : http://whc.unesco.org/fr/list/1256/

33. - Voir : JUPPÉ, Alain. Projet urbain pour la ville de Bordeaux. Bordeaux : Éditions Francom, 1996.

34. - BIDALON, Philippe. "Bordeaux dans dix ans". L'Express, 25 octobre 2007, n² 2938, Supplément spécial, p. XIV.

35. - GRUMBACH, Antoine. «Les bassins à flot : une transition à construire entre centre et lac ». Urbanisme, Hors-série $\mathrm{n}^{\circ} 22$, p. 38.

36. - ESCOLIN, Bertrand. "Un quartier durable aux bassins à flot». Le Moniteur architecture, 5 mars 2010, n 5545, p. 41.

37. - ESCOLIN, Bertrand. "Un quartier durable aux bassins à flot ». Le Moniteur architecture, 5 mars 2010, $\mathrm{n}^{\circ} 5545$, p. 41.

38. - LARÜE-CHARLUS, Michèle. 2030, vers le Grand Bordeaux, une métropole durable. Bordeaux: Direction générale de l'aménagement de la ville de Bordeaux, 2009, p. 89.

\section{RÉSUMÉS}

Active à peine plus d'un an pendant l'Occupation, la base sous-marine de Bordeaux cristallise encore les souvenirs douloureux des heures sombres du Port de la Lune. Le bunker attire pourtant depuis les années 1960 des artistes, cinéastes, plasticiens et musiciens fascinés par l'esprit du lieu. Institutionnalisée au début des années 1990, la base sous-marine devient un espace dédié au nautisme avant d'être consacrée à l'art contemporain au début du XXI ${ }^{\mathrm{e}}$ siècle. Aujourd'hui au cœur du projet d'aménagement des bassins à flot du quartier de Bacalan, la base sous-marine questionne et intrigue ses visiteurs sur son passé, mais aussi - et surtout - sur son avenir.

Build during the German occupation, the submarine base of Bordeaux active during hardly more than a year still crystallizes the painful memories of the dark hours of the "Port de la Lune". Nevertheless, the bunker attracts artists, film-makers, visual artists and musicians fascinated by the spirit of the place since the 1960's. Institutionalized at the beginning of 1990's, the submarine base became a space dedicated to the yachting before contemporary art at the beginning of the $\mathrm{XXI}^{\text {th }}$ century. Nowadays at the heart of the project of redevelopment of the wet docks in the urban area of Bacalan, the submarine base questions and intrigues its visitors on its past, but also - and especially - on its future. 
INDEX

Mots-clés : patrimoine militaire, fortification, XXe siècle, bunker, base sous-marine, Bordeaux, Mur de l'Atlantique, exposition, équipement culturel, patrimonialisation, reconversion architecturale, blockhaus

Keywords : military heritage, fortification, Twentieth century, bunker, submarine base, Bordeaux, Atlantic Wall, exhibition, cultural infrastructure, heritage, architectural reconversion, blockhaus

\section{AUTEUR}

\section{MATHIEU MARSAN}

Titulaire d'un Master Professionnel Régie des CEuvres et Médiation de l'Architecture et du Patrimoine et actuellement étudiant en Master Recherche Histoire de l'art. Université Bordeaux 3 - Michel de Montaigne mathieu.marsan@neuf.fr 